\title{
Role of Pharmacist Intervention in the Management of Anemia Associated with Chronic Kidney Diseases at the Hemodialysis Setting
}

\author{
Bushra Hassan Marouf ${ }^{1 *}$, Intisar Ahmed Yusif ${ }^{2}$, Raad Hassan Najim ${ }^{3}$ \\ 'Department of Pharmacology and Toxicology, College of Pharmacy, University of Sulaimani, Sulaymaniyah, Kurdistan Region, IRAO \\ 2Department of Pharmacology, Kirkuk Medical College, University of Kirkuk, Kirkuk, IRAQ. \\ ${ }^{3}$ Department of Microbiology, Kirkuk Medical College, University of Kirkuk, Kirkuk, IRAQ.
}

\begin{abstract}
Background: The potential impact of pharmacist interventions could improve health outcomes of patients with anemia. However, this attempt has not been documented yet in the developing countries. Aim of the study is to investigate the effect of pharmacist interventions in optimizing management of anemia associated with chronic kidney disease. Methods: An interventional randomized control trial was carried out on 120 anemic patients on hemodialysis for eight months. The impact of the pharmacist's interventions in optimizing anemia management to reach hemoglobin target were investigated. Data were analyzed by GraphPad Prism version 8.2.1. Results: During 16-week period, 1646 interventions and recommendations were performed. The interventions were at the physician, patients, drugs, hospital and administrative level. At the physician level a total of $180(10.9 \%)$ recommendations were proposed, at the drug level, the pharmacist provided 595 (36.1\%) interventions which were mainly related to erythropoietin and iron dose adjustment. At the patient and the hospital level $734(44.6 \%)$ and 137 (8.3\%) interventions have been made respectively. Hemoglobin level increased significantly to $(11.25 \pm 2.29)$ after 16 -weeks in interventional group ( $p$-value $<0.5)$, while
\end{abstract}

in non-interventional group it was increased to $(9.99 \pm 2.54)$ at $16^{\text {th }}$ week although the change was significant compare to the baseline level but it has not reached the target hemoglobin level. In the interventional group $39(65 \%)$ patients reached the target hemoglobin while it was $25(41.6 \%)$ in non-interventional group with a significant difference between both groups ( $p$-value $=0.017$ ). Conclusion: In conclusion the implementation of pharmacist intervention in patients with chronic kidney disease-associated anemia improved hemoglobin level and healthcare outcomes.

Key words: Anemia, Chronic kidney disease, Hemodialysis, Hemoglobin, Pharmacist intervention.

Correspondence

Dr. Bushra Hassan Marouf,

Department of Pharmacology and Toxicology, College of Pharmacy, University of Sulaimani, Sulaymaniyah, 46001, Kurdistan Region, IRAQ.

Phone: +9647701562796

Email: bushra.marouf@univsul.edu.iq

DOI: 10.5530/jyp.2020.12.33

\section{INTRODUCTION}

Anemia is a perennial and serious complication of chronic kidney disease (CKD) that finds during the early stage of the disease and intensify as the kidney function deteriorates. ${ }^{1}$ The $\mathrm{CKD}$-associated anemia is primarily due to the less production of endogenous erythropoietin and some degree of iron deficiency that decreases availability of iron for erythropoiesis. ${ }^{2}$ While its pathophysiology is typically multifactorial, the predominant cause of anemia in patients with chronic kidney disease is failure of the kidneys to produce enough erythropoietin. These patients often suffer from weakness, inability to concentrate, chest pain, fatigue and headache. ${ }^{3}$ Treatment of anemia in CKD is complex and challenging, the increasing number of patients with CKD- associated anemia, insufficient number of nephrologists, the complexity of the disease pathogenesis, hypo responsiveness of Erythropoiesis Stimulating Agents (ESA) therapy, patient's polypharmacy, nonadherence to the medication's regimen and a lack of familiarity with clinical practice guidelines and recommendations for management of anemia, all these are considered as major challenges and barriers to treat anemia in CKD patients. Therefore, multidisciplinary efforts are required to treat CKDassociated anemia and to overcome the barriers of such a complicated condition. The impact of pharmacy services as a part of multidisciplinary efforts to improve outcomes in dialysis patients has been described in many studies. Clinical pharmacists have been demonstrated to alleviate drug-related problems and contribute to improving quality of care in hospitalized patients with many diseases. ${ }^{4}$ They are prepared and trained in therapeutics and actively engaged in implementing and monitoring of therapeutic treatment plans. They are also participated in patient rounds conducting drug history at admission, assisting physicians in analyzing relevant laboratory information and checking medication orders for the plausibility of medication, identifying and solving administration errors, adverse drug reactions and drug-drug interactions. ${ }^{5,4}$ Furthermore, they contribute to improve patient's medication adherence through counseling patients about the purposes and necessity of their prescribed medicines. ${ }^{6}$ The pharmacists' intervention efficiently manages barriers in medication taking thereby support non-adherent patients in improving medication adherence. ${ }^{7}$ Although the clinical pharmacist intervention has implemented in various pharmacist-physician-collaborative practice models such as in diabetes, hypertension and hyperlipidemia, neurological diseases, ${ }^{8}$ rheumatological, ${ }^{9}$ and cardiovascular conditions ${ }^{10}$ and the role of the clinical pharmacist in the dialysis unit has been well established in some developed countries. ${ }^{11}$ However, to our knowledge, the overall level of clinical pharmacist services provided by developing nations is still low $^{12}$ and the potential impact of clinical pharmacist interventions in improvement of clinical status, hematological indices and correction of anemia in dialysis patient in the developing countries including Iraq has not well documented yet, for this purpose the current study has designed to investigate the effectiveness of interventions

This is an open access article distributed under the terms of the Creative Commons Attribution-NonCommercial-ShareAlike 4.0 License, which allows others to remix, tweak, and build upon the work non-commercially, as long as the author is credited and the new creations are licensed under the identical terms. 
carried out by the clinical pharmacists in optimizing management of CKD-associated anemia in the hemodialysis center in Kirkuk HospitalKirkuk City-Iraq.

\section{MATERIALS AND METHODS}

\section{Study Design and Ethical Consideration}

The study was a prospective interventional single-blinded randomized control trial it was carried out for eight months between February 2019 - September 2019 with approval letter of ethical committee of College of Medicine/ University of Suleiman (No\#8 in 2019) at the dialysis centers of Kirkuk General Hospital- Directorate of Health in Kirkuk city; a hospital with 250 beds for hospitalization of chronic kidney disease patients. The study carried out as a two-arm trial (interventional and non-interventional group). All patients gave a written informed consent to participate. Figure 1

\section{Enrollment and Randomization}

Simple randomization using random number table was carried out at the first visit before baseline data collection by the clinical pharmacist. Consenting patients were randomized into either the interventional or non-interventional group. The study included anemic patients $(\mathrm{Hb} \leq 8 \mathrm{~g} /$ $\mathrm{dl}$ ) associated with end stage renal disease with continuous hemodialysis of one to two sessions per week as defined by the United State Kidney Foundation and serum creatinine clearance, glomerular filtration rate tests and met the revised criteria of diagnosis of CKD with anemia. ${ }^{13}$ The patients who were not willing to participate were excluded from the study. One hundred and forty patients were screened for eligibility; 20 patients have been excluded because they were not met the inclusion criteria. The rest (i.e. 120 patients) divided into two groups; first group interventional group of 60 patients and clinical pharmacist services were provided to them, the second group were non-interventional group includes 60 patients they were received usual hospital services and treatment protocol of anemia which was mostly erythropoietin and iron supplement as a part of the current anemia treatment plan without any clinical pharmacist intervention.

\section{Types of Clinical Pharmacist Intervention}

After patient's recruitment, the current study structured a plan based on the previous reports and studies ${ }^{9,14}$ for reviewing their medication, recording the types of clinical pharmacist intervention and patient follow up on regular basis. The main clinical activities that the pharmacist experienced to conduct the present study were; build-up in-hospital guideline for proper use of recombinant human erythropoietin in collaboration with physicians based on international guidelines for treatment of anemia in $\mathrm{CKD},{ }^{15}$ recommending drug information on CKD-associated anemia to physicians and nurses and proposing clinical pharmacist intervention at the physician, drug, patient and hospital level as described in Figure 2. At the baseline visit Hemoglobin ( $\mathrm{Hb}$ ) and ferritin were assessed and serum iron and total iron binding capacity (TIBC) levels to calculate TSAT\% (serum iron x100 divided by total iron binding capacity) were also measured. All the clinical pharmacist services were delivered by a qualified registered pharmacist during ward rounds and direct physician-pharmacist communication in the hemodialysis center and all interventions were recorded and classified in an excel spreadsheet. The categories of clinical pharmacist interventions were based on the previous studies. ${ }^{8}$ The total number of interventions per each month, per each patient and over four months were documented and classified electronically to different level using an excel spreadsheet software to be ready for statistical analysis. Patient's adherence was evaluated by counting the pills, bringing the empty container of the iron therapy. Furthermore, the clinical pharmacist performed verbal

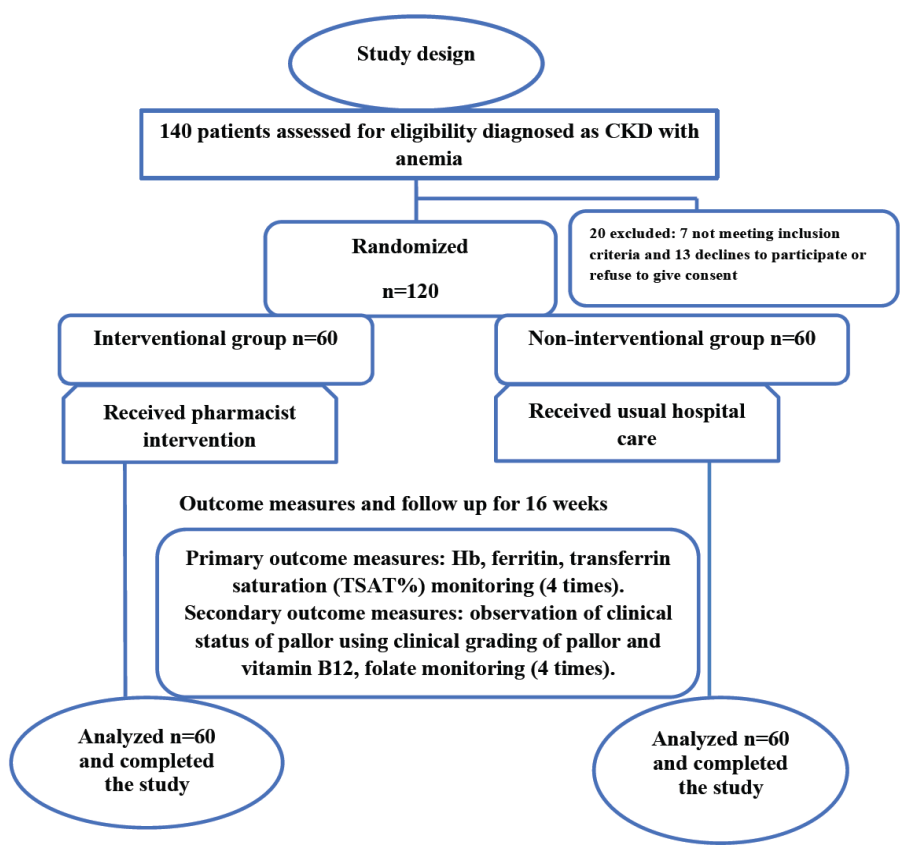

Figure 1: Study design shows randomization and outcome measures. CKD; Chronic kidney disease, TSAT; Transferrin saturation.

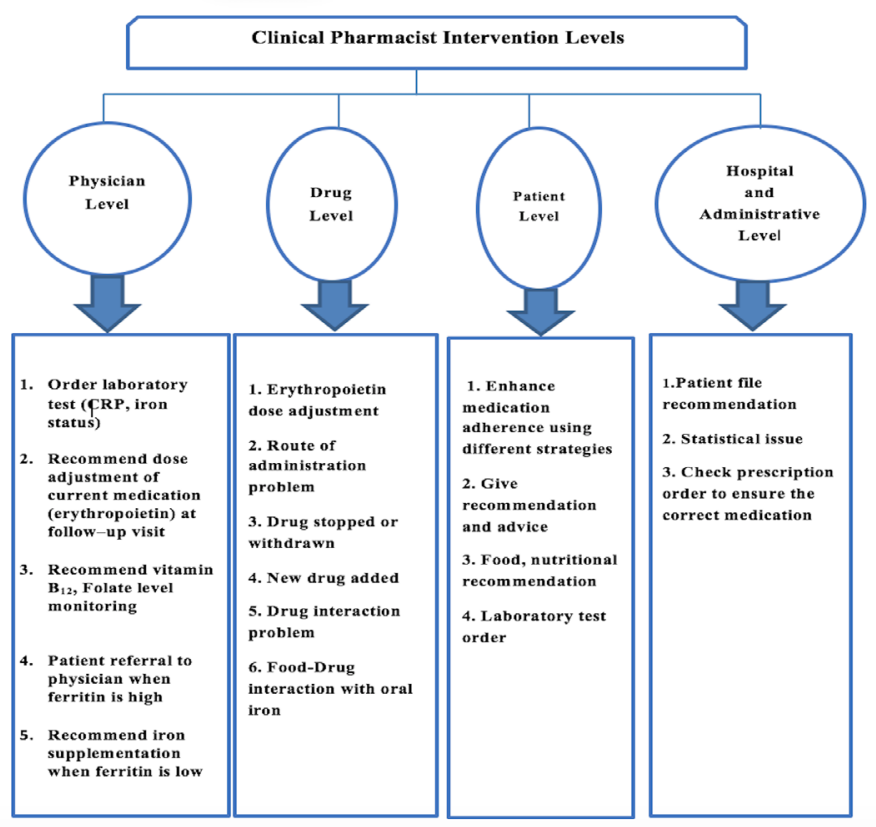

Figure 2: Types of pharmacist's interventions at the physician, drug, patient and hospital-administrative level (Structured and adapted). ${ }^{8,17}$, CRP;C-reactive protein.

recommendations to physicians with regard to interventional group management plan including initiation of iron therapy and appropriate starting doses. Moreover, on each follow up visit patients in interventional group were verbally counselled regarding ESA, iron therapy and importance of anemia management, modification of diet, drug-food and drug-drug interactions, treatment recommendations and adverse effect of the medications. At the baseline visit the patient was referred to a physician by the clinical pharmacist according to recommendations adapted from cases et al. and Ohnishi J et al. ${ }^{16,17}$ All patients in the 
interventional group were followed up by the clinical pharmacist on a periodic weekly visit to the dialysis setting and through phone calls. The clinical pharmacist role was to remind patients prior to their scheduled follow-up appointment. Patients in the non-interventional group received the usual medical care by their physician without the clinical pharmacist intervention.

\section{Primary outcome measure}

The main outcomes were the impact of the pharmacist involvement in optimizing anemia management, investigating hematological indices such as hemoglobin, serum iron and TIBC, ferritin, transferrin saturation (TSAT\%) to reach or maintain hemoglobin within target range (10 -11.5 $\mathrm{g} / \mathrm{dL}$ or $10-12 \mathrm{~g} / \mathrm{dL})^{18}$ and this was performed by regular follow up starting from $1^{\text {st }}$ week (baseline) to $4^{\text {th }}$ weeks, $8^{\text {th }}$ weeks, $12^{\text {th }}$ weeks and $16^{\text {th }}$ weeks and comparing with the non-interventional group.

\section{Secondary outcome measure}

Includes clinical status monitoring using clinical grading of pallor; a researcher sequentially examined conjunctiva, tongue, skin, palm andnail bed of each studied patient. Based on the clinical grading of pallor the physical status of the patients classified into: Mild: pallor of conjunctiva and/ or mucous membrane. Moderate: pallor of conjunctiva and/or mucous membrane + pallor of skin. Severe: pallor of conjunctiva and/or mucous membrane + pallor of skin + pallor of palmar creases.${ }^{19}$ Furthermore, as a secondary outcome measure vitamin $\mathrm{B}_{12}$ and folate levels, $\mathrm{C}$-reactive protein (CRP); to identify factual ferritin level, complete blood picture (CBP) have also been measured and compared with the baseline values and the non-interventional group.

\section{Statistical analysis}

Data were analyzed utilizing GraphPad Prism version 8.2.1, chi-square (Fisher's exact test) was used to compare the percentage of patients reaching their primary goal of $\mathrm{Hb}$ between the interventional and noninterventional groups. A paired sample t-test was used to compare initial and follow-up hematological indices' means within each group. An independent sample $t$-test was used to compare the change in patient's hematological indices' means between the interventional and non-interventional groups. A $p$-value $\mathfrak{£} 0.05$ was considered statistically significant.

\section{RESULTS}

Basic characteristic of the participants shows in Table 1. The mean age for the interventional group was $49.43 \pm 14.62$ years while it was $51.58 \pm 17.76$ years for the non-interventional group with a $p$-value $=0.47$, non-significant difference was observed between male and female patients ( $p$-value $=0.86$ ), nearly other demographic data including body weight, occupation, number of dialysis sessions per week were matched at the screening and baseline visit and there were non-significant differences between interventional and non-interventional groups. Percentage of patients with normocytic and normochromic type

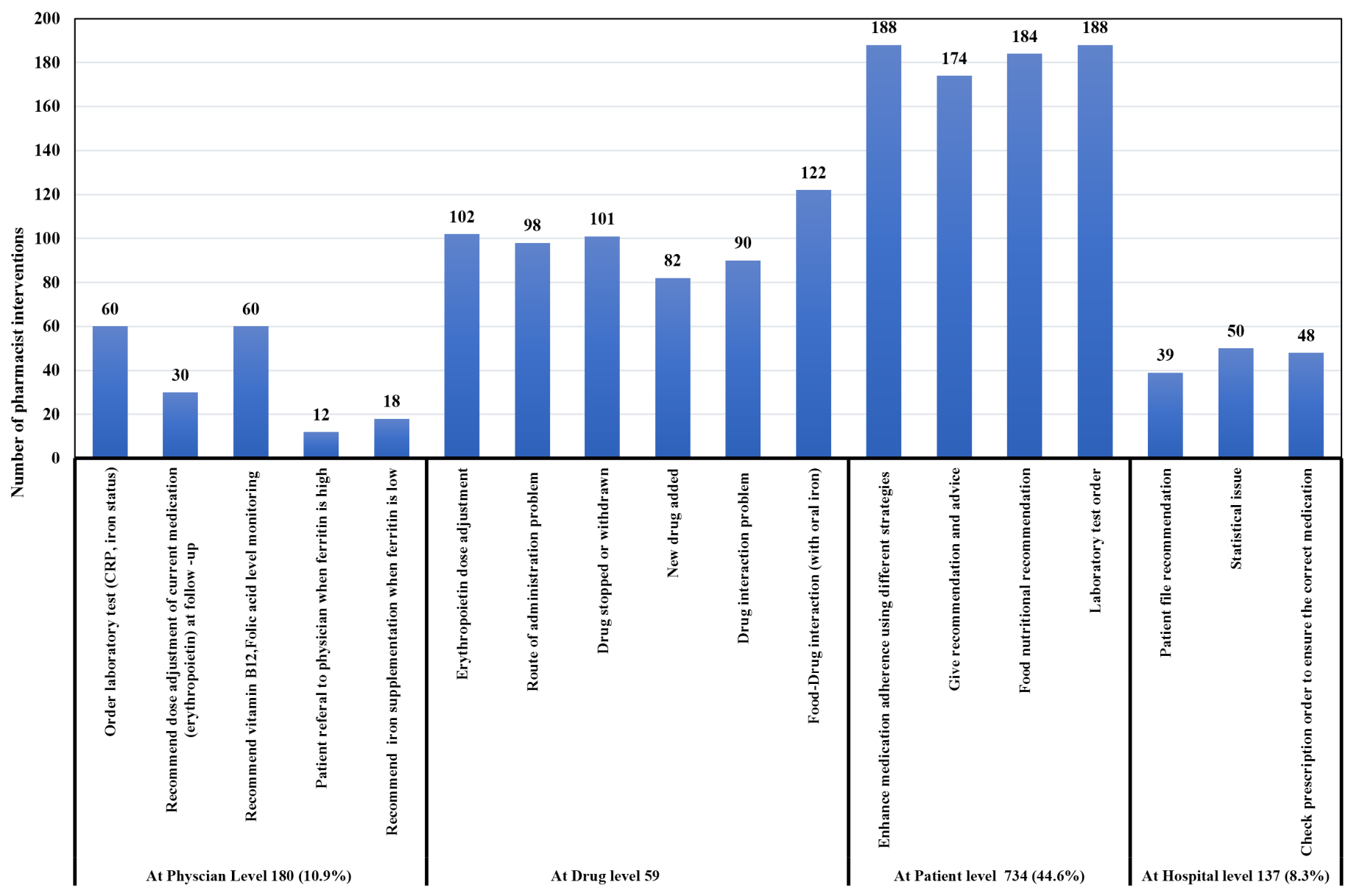

Figure 3: Types of pharmacist interventions and recommendations at physician, drugs, patients, hospital and administrative level during 16-week period. Values expressed as number and percentage. CRP; C-reactive protein. 
[(ferritin $>100 \mathrm{ng} / \mathrm{ml}$ and transferrin saturation index [TSAT], >20\%)] of anemia in interventional and non-interventional group were $70 \%$ and $71.67 \%$ respectively, meanwhile those with IDA (hypochromic microcytic) in both groups were $30 \%$ in interventional and $28.33 \%$ in non-interventional ( $p$-value=0.9). Patients in both groups were found to have a comparable history of comorbid diseases such as hypertension and diabetes 34 (56.6\%) in interventional group, while $36(60 \%)$ in noninterventional group with $p$-value $=0.87$.

Table 1: Demographic data and basic characteristic of the patients participated in the study $n=120$.

\begin{tabular}{|c|c|c|c|}
\hline & \multicolumn{2}{|c|}{ Group } & \multirow[t]{2}{*}{$P$-value } \\
\hline & $\begin{array}{l}\text { Intervention } \\
\quad(n=60)\end{array}$ & $\begin{array}{l}\text { Non-intervention } \\
\qquad(n=60)\end{array}$ & \\
\hline Age; Mean \pm SD (year) & $49.43 \pm 14.62$ & $51.58 \pm 17.76$ & 0.471 \\
\hline \multicolumn{4}{|c|}{ Gender n(\%) } \\
\hline Male & $32(53.3)$ & $33(55.0)$ & \\
\hline Female & $28(46.7)$ & $27(45.0)$ & 0.86 \\
\hline Body weight $(\mathrm{Kg})$ & $62.93 \pm 1.49$ & $62.78 \pm 1.59$ & 0.945 \\
\hline \multicolumn{4}{|c|}{ Occupation $\mathrm{n}(\%)$} \\
\hline Not working & $58(96.7)$ & $60(100.0)$ & \\
\hline Working & $2(3.3)$ & $0(0.0)$ & 0.496 \\
\hline $\begin{array}{l}\text { Duration of disease } \\
\text { (year) }\end{array}$ & $2.77 \pm 0.12$ & $2.39 \pm 1.69$ & 0.06 \\
\hline \multicolumn{4}{|c|}{ Number of dialysis session / Week } \\
\hline 1.0 & $37(61.7)$ & $33(55.0)$ & \\
\hline 2.0 & $23(38.3)$ & $27(45.0)$ & 0.4 \\
\hline \multicolumn{4}{|c|}{ Patients with Hypertension and Diabetes n(\%) } \\
\hline Yes & $34(56.6 \%)$ & $36(60 \%)$ & \\
\hline No & $26(43.4 \%)$ & $24(40.0 \%)$ & 0.87 \\
\hline \multicolumn{4}{|c|}{ Patients with other comorbidities $\mathrm{n}(\%)$} \\
\hline Yes & $17(28.3 \%)$ & $18(30 \%)$ & \\
\hline No & $43(71.6 \%)$ & $42(70 \%)$ & 0.7 \\
\hline
\end{tabular}

Values are presented as percent or mean $\pm \mathrm{SD}$; $n$ : number of patients. Independent -sample $t$-test and Chi-square test for non-parametric variables were utilized to predict significance at $P<0.05$.SD; Standard deviation.

\section{Types of pharmacist interventions}

During the 16-weeks study period, the pharmacist interacted and reviewed the medication files for 60 admitted patients every visits. Of the total patients, 1646 interventions and recommendations were carried out. The interventions were at the physician, patients, drugs, hospital and administrative level as shown in Figure 3. At the physician level a total of $180(10.9 \%)$ recommendations and interventions were proposed by

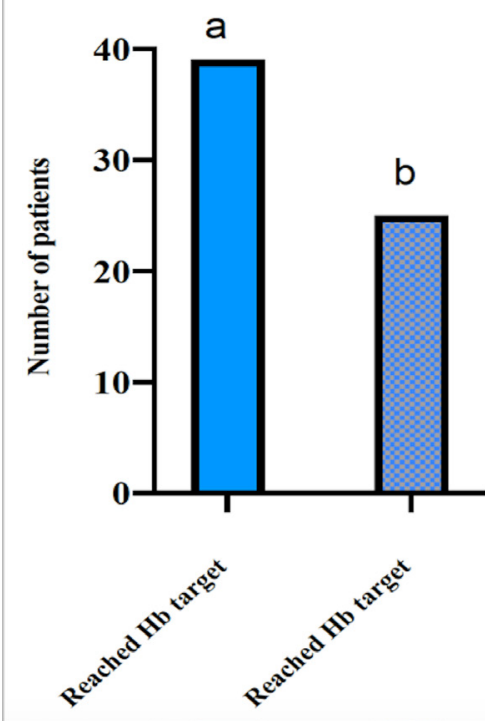

Figure 4: Patients reached hemoglobin target in interventional and non-interventional group after16-weeks period $n=120$. Values are expressed as number, chi-square (Fisher's exact test) was used to compare different group and predict significance at $p<0.05$. Non identical letters $(a, b)$ indicates significantly different between both studied group. Hb;hemoglobin.

Table 3: Patients with declined or unchanged $\mathrm{Hb}$ level in interventional and non-interventional group $n=120$.

\begin{tabular}{cccc}
\hline Criteria & $\begin{array}{c}\text { Interventional } \\
\text { group } n=60\end{array}$ & $\begin{array}{c}\text { Non- } \\
\text { interventional } \\
\text { group } n=60\end{array}$ & p-value \\
\hline $\begin{array}{c}\text { Patients who had a decline } \\
\text { in their Hb or remained } \\
\text { unchanged }\end{array}$ & $5(8.3 \%)$ & $17(28.3 \%)$ & 0.008 \\
\hline
\end{tabular}

Values are expressed as number and percent, chi-square (Fisher's exact test) was used to compare different group and predict significance at $p<0.05$; Hb;hemoglobin.

Table 2: Hematological indices in interventional and non-interventional group during 16-weeks period $n=120$.

\begin{tabular}{|c|c|c|c|c|c|c|c|c|}
\hline \multirow{2}{*}{$\begin{array}{c}\text { Hematological } \\
\text { parameter }\end{array}$} & \multicolumn{4}{|c|}{ Interventional group $(n=60)$} & \multicolumn{4}{|c|}{ Non-interventional group $(n=60)$} \\
\hline & $\begin{array}{c}1^{\text {st }} \text { week } \\
\text { (Baseline) }\end{array}$ & $4^{\text {th }}$ week & $8^{\text {th }}$ week & $16^{\text {th }}$ week & $\begin{array}{c}1^{\text {st }} \text { week } \\
\text { (Baseline) }\end{array}$ & $4^{\text {th }}$ week & $8^{\text {th }}$ week & $16^{\text {th }}$ week \\
\hline $\mathrm{Hb} \mathrm{g/dl}$ & $7.64 \pm 0.45 \mathrm{a}$ & $8.34 \pm 0.98 * b c$ & $10.09 \pm 1.72^{*} \mathrm{~d}$ & $11.25 \pm 2.29^{*} \mathrm{a}$ & $7.28 \pm 0.80$ & $8.40 \pm 1.21^{*} b$ & $9.93 \pm 2.37^{*} \mathrm{c}$ & $9.99 \pm 2.54^{*}$ \\
\hline TSAT $\%$ & $40 \pm 7.05$ & $41 \pm 0.12^{c}$ & $45 \pm 0.14^{b}$ & $47 \pm 0.17^{\text {ad }}$ & $38 \pm 0.10^{\mathrm{b}}$ & $39 \pm 0.11^{\mathrm{b}}$ & $48 \pm 0.12^{* c}$ & $40 \pm 0.13^{\mathrm{d}}$ \\
\hline S.Ferritin $\mathrm{ng} / \mathrm{ml}$ & $419.02 \pm 445.31$ & $289.96 \pm 332.65^{\star}$ & $229.83 \pm 40.53^{*}$ & $248.75 \pm 67.93^{*}$ & $536.45 \pm 439.17$ & $272.61 \pm 309.76^{*}$ & $234.99 \pm 272.51^{*}$ & $275.48 \pm 309.18^{*}$ \\
\hline S. Vitamin $B_{12} \mathrm{ng} / \mathrm{ml}$ & $289.03 \pm 113.83$ & $296.20 \pm 114.28$ & $287.20 \pm 112.22$ & $327.70 \pm 26.10$ & $297.63 \pm 120.45$ & $295.22 \pm 114.59$ & $286.83 \pm 111.50$ & $244.50 \pm 111.10$ \\
\hline S.Folate $\mathrm{ng} / \mathrm{ml}$ & $13.09 \pm 4.39$ & $12.03 \pm 3.11 \mathrm{~b}$ & $13.66 \pm 4.12 \mathrm{~d}$ & $12.56 \pm 3.06$ & $14.39 \pm 3.94$ & $11.90 \pm 3.06^{*} \mathrm{~b}$ & $13.58 \pm 4.12^{*} \mathrm{~d}$ & $12.47 \pm 3.12^{\star}$ \\
\hline
\end{tabular}

Values are presented as mean \pm S.D; $n$ : number of patients; ${ }^{*}$ significantly different compared with baseline values within the same group (paired $t$-test); values with superscripts (a) are significantly different between different groups (independent $t$-test a; $P<0.05$ ). while values with superscripts $(\mathrm{b}, \mathrm{c}, \mathrm{d})$ are significantly different among different times within the same group. Hb;hemoglobin, TSAT; Transferrin saturation. 
Table 4: Clinical grade of pallor of the patients in interventional group with non-interventional group over the period of follow up $n=120$.

\begin{tabular}{|c|c|c|c|}
\hline Time & Clinical Grade & Interventional group $n=60(\%)$ & Non-interventional group $n=60(\%)$ \\
\hline \multirow[t]{2}{*}{$1^{\text {st }}$ month (Baseline) } & Mild & $1(1.7)$ & $1(1.7)$ \\
\hline & Moderate & $48(80.0)$ & $46(76.7)$ \\
\hline \multirow[t]{2}{*}{$2^{\text {nd }}$ month } & Mild & $1(1.7)$ & $1(1.7)$ \\
\hline & Moderate & $52(86.7)$ & $46(76.7)$ \\
\hline \multirow{2}{*}{$3^{\text {rd }}$ month } & Moderate & $36(60.0)$ & $46(76.7)$ \\
\hline & Severe & $7(11.7)$ & $13(21.7)$ \\
\hline \multirow[t]{2}{*}{$4^{\text {th }}$ month } & Mild & $35(58.3)$ & $4(6.7)$ \\
\hline & Moderate & $17(28.3)$ & $49(81.7)$ \\
\hline
\end{tabular}

Values expressed as number and percentage

the pharmacist to optimize management of anemia. The most common interventions were interaction with physician to order laboratory monitoring to identify frequent hypo responsiveness to ESA resulting from various condition such as iron deficiency and inflammation, further to highlight the association between serum ferritin as an iron storage molecule and as an acute phase reactant protein. Almost all of the recommendations proposed by the pharmacists were accepted by the physician. At the drug level, the pharmacist provided 595 (36.1\%) interventions which were mainly related to erythropoietin dose adjustment, route of administration complaint that required changing drug formulation, drug stopped or withdrawn and food-drug interaction with oral iron. At the patient level also 734 (44.6\%) interventions have been made by the pharmacist including patient's recommendations to improve medication adherence using different strategies, food and nutritional recommendation and laboratory order for all patients and follow up. Furthermore, the pharmacist also interacted with the administrative department of the hospital for documentation of the statistical affair and patient file recommendation as well as procurement decision. The total number of interventions carried out by the pharmacist at the hospital and administrative level was 137 (8.3\%). All the pharmacist interventions were exclusively applied to the interventional group during the study period.

\section{Hematological parameters Hemoglobin}

Hemoglobin level at the baseline was $(7.64 \pm 0.45)$ in interventional group in compare to $(7.28 \pm 0.80)$ in non-interventional group, both groupswerehaving severeanemia.Hemoglobinlevelincreasedsignificantly to $(11.25 \pm 2.29)$ after 16 -weeks in interventional group ( $p$-value $<0.5)$ while in non-interventional group it was increased to $(9.99 \pm 2.54)$ at $16^{\text {th }}$ week although the change was significant compare to the baseline level but it has not reached the target Hb level (Table 2). After 16-weeks period of follow-up and pharmacist intervention provision, 39 (65\%) patients of the interventional group reached the target $\mathrm{Hb}$ while in non-interventional group it was $25(41.6 \%)$ with a statistically significant difference between both groups ( $p$-value $=0.017$ ) as shown in Figure 4 . In interventional group only $5(8.3 \%)$ has declined or unchanged $\mathrm{Hb}$ while $17(28.3 \%)$ patients in non-interventional group their $\mathrm{Hb}$ level was declined or unchanged with p-value of 0.008 (Table 3).

\section{Iron metabolism indices}

The transferrin saturation (TSAT\%) was $(40 \pm 7.05) \%$ at the baseline in interventional group and it was $(47 \pm 0.17) \%$ at week 16 in comparison to baseline value of $(38 \pm 0.10) \%$ in regard to non-interventional group in which the TSAT\% was slightly increased to $(40 \pm 0.13) \%$. Serum ferritin baseline value of patients undergo intervention was $(419.02 \pm 445.31)$ $\mathrm{ng} / \mathrm{ml}$, after 16 weeks the level was seen to be $(248.75 \pm 267.93) \mathrm{ng} / \mathrm{ml}$, higher changes was observed in those patients in non-interventional group without pharmacist services as their baseline level of ferritin was $(536.45 \pm 439.17) \mathrm{ng} / \mathrm{ml}$ which became $(275.48 \pm 309.18) \mathrm{ng} / \mathrm{ml}$ after 16 weeks as shown in Table 2. Mean value of serum vitamin $B_{12}$ was $(289.03 \pm 113.83) \mathrm{ng} / \mathrm{ml}$ and $(297.63 \pm 120.45) \mathrm{ng} / \mathrm{ml}$ for interventional and non-interventional group respectively at the baseline, at the end of the study non-significant changes between both groups were observed; $(327.70 \pm 126.10) \mathrm{ng} / \mathrm{ml}$ and $(244.50 \pm 111.10) \mathrm{ng} / \mathrm{ml}$ respectively (Table 2).

As a secondary outcome measure physical examination was done for all anemic patients and clinical grading of pallor has been recorded on a monthly basis, Table 4 shows the comparison between patients in interventional group with non-interventional group in regard to clinical status over the period of follow up, a remarkable improvement was seen at $12^{\text {th }}$ and $16^{\text {th }}$ weeks with those patients under intervention. At the baseline only $1(1.7 \%)$ patient was in mild status, while the rest were either with moderate or severe grade of pallor. At $12^{\text {th }}$ and $16^{\text {th }}$ weeks number of patients with mild grade of pallor has increased to $17(28.3 \%)$ and $35(58.3 \%)$ respectively with decrement of patients in moderate and severe categories. While non-interventional group shows unnoticeable improvement toward mild clinical status of pallor with a slight change between baseline and last two months of follow-up in moderate to severe pallor of conjunctiva and/or mucous membrane and skin.

\section{DISCUSSION}

The principle finding of the current study showed that the clinical pharmacy services resulted in a significant improvement in $\mathrm{Hb}$ level in the interventional group and greater number of patients in interventional group achieved their subnormal target $\mathrm{Hb}$ compared with noninterventional group. Previous reports and evidences favored subnormal level of $\mathrm{Hb}(10-11.5 \mathrm{~g} / \mathrm{dL})$ or $(10-12 \mathrm{~g} / \mathrm{dL})$ in patients with $\mathrm{CKD}$, while the total correction of anemia $(\mathrm{Hb} \geq 13 \mathrm{~g} / \mathrm{dL})$ during administration of ESA is not preferred, as this level is associated with deleterious effect such as stroke, cardiovascular risks, thrombosis, elevated blood pressure, cancer progression. ${ }^{18}$ This improvement in $\mathrm{Hb}$ level in the current study is partly 
Marouf, et al:: Impact of Pharmacy Practice in Correction of Anemia in Patients with End Stage Renal Disease

related to the enhancement of patient's adherence to their medications by efforts of the pharmacist using different strategies. On the other hand, it might have related to extensive and continuous monitoring of the patient's laboratory investigation, recommendation on food and life style modification by the clinical pharmacist as well as by providing many interventions which were mostly circulate around erythropoietin and elemental iron dose adjustment. The current finding is consistent with the study done by Bucaloiu et al. in which significant higher percentage of patients achieving hemoglobin and transferrin saturation values target range through a pharmacist-managed program..$^{20}$

Failure to detect iron deficient patient among CKD-anemic patients can have deleterious consequences. In the present study the pharmacist has measured iron status in conjunction with $\mathrm{Hb}$ in all participants to ensure that the patients have adequate iron stores when initiating ESA therapy as well as to add iron supplementation simultaneously with ESA once the patient has inadequate iron level. Iron deficiency can attenuate the response to ESAs. Therefore, iron supplementation is now a recognized treatment of renal anemia. ${ }^{21}$ The most conventional blood iron indices that have been measured in this study were serum ferritin and STAT. Ferritin reflects iron stores of the body, it is an acute-phase reactant and its levels increase in response to inflammation, which is usually happens in many patients undergoing hemodialysis and it may also be elevated in iron-deficient patients due to malnourishment, malignancy and infection. ${ }^{22}$ In the present study patients with either low or high ferritin level have been monitored and accordingly treatment protocol has been manipulated and recommended by the pharmacist within the multidisciplinary team.

The pharmacist in the present study focused on identifying of the cases of ESA hypo responsiveness. The two major factors accounting for the state of hypo responsiveness are iron deficiency and inflammation. ${ }^{23}$ Therefore, in the present study the pharmacist performed regular monthly monitoring of iron status and numerous interventions were done in regard to iron supplementation, erythropoietin dose adjustment and advising the patients to adhere to their medications. Pharmacists provided drug information to physicians, suggested therapeutic plans, evaluated medication use and educated patients regarding their medications and lifestyle modifications. Our study was in line with the previous effort which was done to optimize $\mathrm{Hb}$ level after three month-implementation of a pharmacist's educational program for hemodialysis patients. ${ }^{24}$ Evaluation of vitamin $\mathrm{B}_{12}$ and folate levels are considered as a part of standard components during diagnosis of anemia, no much differences were observed in the current study on both vitamin $B_{12}$ and folate between both groups at the baseline and after 16 weeks of intervention, these results could be due to that the cause of anemia in chronic kidney disease mainly is the inadequate production of endogenous erythropoietin, iron deficiency or functional anemia. Although a recent review also considers folic acid and/ or vitamin $B_{12}$ supplementation as appropriate adjunctive therapy in patients with CKD. ${ }^{25}$ Furthermore the obtain results of clinical examination in the present study exhibited a noticeable improvement in the paleness of the skin and conjunctival pallor in interventional group compare to the non-interventional group this finding attributed to the critical role of pharmacist in assisting the patients to adhere to their medication, particularly in areas of iron supplementation, dietary advice and recommendation, food and/or drug interaction with oral iron. This finding is consistent with result reported by Kucera who stated that the pharmacist has a critical role in the treatment of patients with anemia. ${ }^{26}$ The current study was not without limitations such as a relatively small sample size, conducting the study in a single hemodialysis center preclude generalization of the results to other centers, additionally contamination of the patients in interventional with non-interventional group results in non-exclusive provision of pharmacist services to interventional group and may lead to bias in the outcomes. However, the strong side of the current study is that it was a first clinical trial that documented the association between pharmacist intervention in regards to correction of anemia in a hemodialysis center in Iraq.

\section{CONCLUSION}

In conclusion the implementation of pharmacist intervention in patients with CKD-associated anemia undergoing hemodialysis improved hemoglobin level and healthcare outcomes.

\section{ACKNOWLEDGEMENT}

The authors appreciate the kind support of the college of PharmacyUniversity of Sulaimani and cooperation of the hemodialysis center in Kirkuk Hospital. The authors would like to thank the patients who are participated in the study.

Funding

This study was self-funded.

\section{CONFLICT OF INTEREST}

The authors declare no conflict of interest.

\section{ABBREVIATIONS}

TSAT: Transferrin saturation; ESA: Erythropoiesis stimulating agents.

\section{SUMMARY}

In summary the provision of clinical pharmacist's interventions and recommendations to patients with $\mathrm{CKD}$-associated anemia contributes to the optimization of pharmacotherapy and improved hemoglobin level to reach target level and consequently improvement of clinical status outcomes of the patients. The study recorded large numbers of pharmacist interventions at physicians, drugs, patients and hospital level.

\section{REFERENCES}

1. Ryu S, Park S, Jung J, Kim Y, Oh Y, Yoo T, et al. The prevalence and management of anemia in chronic kidney disease patients: result from the Korean Cohort Study for Outcomes in Patients with Chronic Kidney Disease (KNOW-CKD). J Korean Med Sci. 2017;32(2):24956.

2. Babitt JL, Lin HY. Mechanisms of anemia in CKD. J Am Soc Nephrol 2012;23(10):1631-4

3. Fishbane S, Spinowitz B. Update on Anemia in ESRD and Earlier Stages of CKD: Core Curriculum 2018. Am J Kidney Dis. 2018;71(3):423-35

4. Yi Z, Sun S, Li X, Lu M, Zhai S. An evaluation of clinical pharmacist service on a neurology care unit. Int J Clin Pharm. 2016;38(1):30-3.

5. Lenssen R, Heidenreich A, Schulz J, Trautwein C, Fitzner C, Jaehde U, et al. Analysis of drug-related problems in three departments of a German University hospital. Int J Clin Pharm. 2016;38(1):119-26.

6. Debenito J, Billups S, Tran T, Price L. Impact of a Clinical Pharmacy Anemia Management Service on Adherence to Monitoring Guidelines, Clinical Outcomesand Medication Utilization. J of Manag Care spec Pharm. 2014;20(7):715-20

7. Zwikker H, DenBemt BV, DenEnde CV, Lankveld WV, Broeder A, DenHoogen $\mathrm{FV}$, et al. Development and content of a group-based intervention to improve medication adherence in non-adherent patients with rheumatoid arthritis. Patient Educ Couns. 2012;89(1):143-51.

8. Ali M, Khedr E, Ahmed F, Mohamed N. Clinical pharmacist interventions in managing drug-related problems in hospitalized patients with neurological diseases. Int J Clin Pharm. 2018;40(5):1257-64.

9. Yailian A, Revel E, Tardy C, Fontana A, Estublier C, Decullier E, et al. Assessment of the clinical relevance of pharmacists' interventions performed during medication review in a rheumatology ward. Eur J Intern Med. 2019;59:91-6.

10. Jiang SP, Chen J, Zhang XG, Lu XY, Zhao OW. Implementation of pharmacists' interventions and assessment of medication errors in an intensive care unit of a Chinese tertiary hospital. Ther Clin Risk Manag. 2014;10:861.

11. Al Raiisi F, Stewart D, Fernandez-Llimos F, Salgado T, Mohamed M, Cunningham S. Clinical pharmacy practice in the care of Chronic Kidney Disease patients: a systematic review. Int J Clin Pharm. 2019;41(3):630-666. 
12. Abduelkarem AR. Extending the role of pharmacists in patient care: Are pharmacists in developing nations ready to change?. Pharmacol Pharm. 2014;5:865-75.

13. KDIGO 2012. Clinical Practice Guideline for the Evaluation and Management of Chronic Kidney Disease. Kidney Int Suppl. 2013;3:1-136.

14. StPeter W, Wazny L, Patel U. New models of chronic kidney disease care including pharmacists: Improving medication reconciliation and medication management. Curr Opin Nephrol Hypertens. 2013;22(6):656-62.

15. Mikhail A, Brown C, Williams J, Mathrani V, Shrivastava R, Evans J, et al. Renal association clinical practice guideline on Anemia of Chronic Kidney Disease. BMC Nephrology. 2017;18(1):345.

16. Cases A, Egocheaga M, Tranche S, Pallarés V, Ojeda R, Górriz J, et al. Anemia of chronic kidney disease: Protocol of study, management and referral to Nephrology. Nefrología. 2018;38(1):8-12.

17. Ohnishi J, Miyake A, Kuwatsuka K, Onoue Y, Lee M, Koyama T, et al. Effect of Pharmacist Management on Serum Hemoglobin Levels with Renal Anemia in Hemodialysis Outpatients. Biol Pharm Bull. 2011;34(10):1609-12.

18. Nakhoul G, Simon J. Anemia of chronic kidney disease: Treat it, but not too aggressively. Cleve Clin J Med. 2016;83(8):613-24.

19. Gupte S. Differential Diagnosis in Pediatrics- $5^{\text {th }}$ edition. Jaypee Publishers. 2008;90-1.
20. Bucaloiu ID, Akers G, Bermudez MC, Mainali R, Brown BL, Roberts SS, et al Outpatient erythropoietin administered through a protocol-driven, pharmacistmanaged program may produce significant patient and economic benefits. Manag Care Interface. 2007;20(6):26-30.

21. Pergola P, Fishbane S, Ganz T. Novel Oral Iron Therapies for Iron Deficiency Anemia in Chronic Kidney Disease. Adv Chronic Kidney Dis. 2019;26(4):272-91.

22. Wong M, Tu C, LiY, Perlman R, Pecoits-Filho R, Lopes A, et al. Anemia and iron deficiency among chronic kidney disease Stages 3-5ND patients in the Chronic Kidney Disease Outcomes and Practice Patterns Study: Often unmeasured, variably treated. Clin Kid Journal. 2019.

23. Stenvinkel P.The role of inflammation in the anaemia of end-stage renal disease. Nephrol Dial Transplant. 2001;16(suppl 7):36-40.

24. Allenet B, Chen C, Romanet T, Vialtel P, Calop J. Assessing a pharmacist-run anemia educational program for patients with chronic renal insufficiency. Pharmacy World and and Science. 2007;29(1):7-11.

25. Capelli I, Cianciolo G, Gasperoni L, Zappulo F, Tondolo F, Cappuccilli M, et al. Folic Acid and Vitamin $B_{12}$ Administration in CKD, Why Not?. Nutrients. 2019;11(2):383.

26. Kucera A, Smith B, Tsu LV. Anemia and the Role of the Pharmacist. Arizona Journal of Pharmacy 2014; 31-35.

Article History: Submission Date : 27-12-2019; Revised Date : 18-03-2020; Acceptance Date : 06-04-2020.

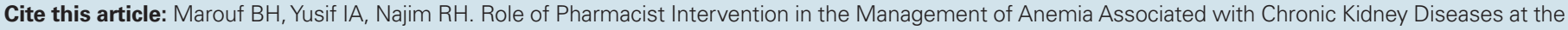
Hemodialysis Setting. J Young Pharm. 2020;12(2):162-8. 